\title{
Accumulation of genomic aberrations during clinical progression of medulloblastoma
}

\author{
Andrey Korshunov • Axel Benner • Marc Remke • \\ Peter Lichter $\cdot$ Andreas von Deimling $\cdot$ Stefan Pfister
}

Received: 14 July 2008 / Revised: 6 August 2008 / Accepted: 6 August 2008 / Published online: 15 August 2008

(C) The Author(s) 2008. This article is published with open access at Springerlink.com

\begin{abstract}
Medulloblastomas comprise the most frequent malignant brain tumor in childhood and one of the biggest challenges in pediatric oncology. The current concept suggests that these tumors may undergo stepwise progression as it has been shown for other brain tumors. However, conclusive evidence of molecular progression over time has not been demonstrated yet for medulloblastoma. In the present study, 28 pairs of medulloblastoma at primary diagnosis and at the time of recurrence, either occurring as local tumor regrowth or tumor dissemination, were histopathologically and molecularly analyzed. Cytogenetic analysis included interphase fluorescence in situ hybridization for five genomic loci (MYC, MYCN, 17p, 17q, 6q) that have previously been identified as prognostic markers in primary tumors. Of 16 tumors showing early recurrence $(<4$ years after first diagnosis), only one showed increased histological
\end{abstract}

A. Korshunov $\cdot$ A. von Deimling

Clinical Cooperation Unit Neuropathology,

German Cancer Research Center, Im Neuenheimer Feld 280,

69120 Heidelberg, Germany

\section{A. Korshunov $(\varangle) \cdot$ A. von Deimling}

Department of Neuropathology, University of Heidelberg, Im Neuenheimer Feld 220/221, 69120 Heidelberg, Germany e-mail: Andrey.Korshunov@med.uni-heidelberg.de

\author{
A. Benner \\ Division Biostatistics, German Cancer Research Center, \\ Im Neuenheimer Feld 280, 69120 Heidelberg, Germany \\ M. Remke $\cdot$ P. Lichter $\cdot$ S. Pfister \\ Division Molecular Genetics, German Cancer Research Center, \\ Im Neuenheimer Feld 280, 69120 Heidelberg, Germany

\section{S. Pfister} \\ Department of Pediatric Oncology, Hematology \& Immunology, \\ University of Heidelberg, Im Neuenheimer Feld 153, \\ 69120 Heidelberg, Germany
}

anaplasia in the secondary lesion (6\%), and two acquired genomic lesions indicative for a more malignant phenotype (13\%). In contrast to this, of 12 tumors with a time to recurrence of 4 years or more, nine tumors $(75 \%)$ showed a more malignant phenotype either reflected by increased anaplasia alone or by both increased anaplasia and acquirement of genomic aberrations known to be associated with inferior patient outcome. These results suggest that early recurrence in medulloblastoma mainly occurs in tumors with a highly malignant genotype and phenotype per se, whereas late recurrence is often dependent on tumor evolution toward a more malignant biology. Therefore, biopsy of recurrent tumors should be performed to assess the biologic properties of the relapsed tumor, especially when targeted therapy approaches are considered.

Keywords Medulloblastoma $\cdot$ Progression - Metastasis . Genomic aberration $\cdot$ Anaplasia $\cdot$ Chromosome $17 q$

\section{Introduction}

Brain tumors are the leading cause of cancer-related mortality in pediatric patients, with medulloblastoma (MB) being the most common representative in this age group. Considerable advances have been made in the treatment of MB in the last three decades by using multimodal therapeutic regimens involving surgery, radiotherapy, and chemotherapy. About $10-15 \%$ of patients die from disease within 2 years after diagnosis, whereas approximately $60 \%$ of patients can nowadays be cured [9, 11, 22, 23]. However, the predictive performance of current risk stratification, which is entirely based on clinical variables, needs to be improved because of the dismal prognosis of children with tumor relapse, and because of relevant treatment-induced 
long-term effects of survivors [21]. Clinical staging systems based on age, metastatic stage at diagnosis and, in some studies, extent of surgical resection have so far been the most useful methods for the stratification of patients into standard and high risk therapy groups $[1,26,27]$. Additionally, after refinement of the histological classification of MB in the 2007 WHO classification, the histological subtype is of potential prognostic relevance: large cell/anaplastic MB behaves more aggressively, while nodular/ desmoplastic variants show a more favorable clinical course as compared to the large group of classic MB [3, 5 , $7,10,13,14,17]$. Some recent studies have extended the concept of anaplasia in MB and suggested that this phenomenon reflects malignant progression of a tumor and may occur in various MB subtypes independent of large cell histology $[3,4,12]$. Progression over time from nonanaplastic to anaplastic MB has been described in cases with local tumor recurrence $[3,12]$ as well as in secondary metastatic lesions with or without local recurrence [4].

To date, numerous molecular studies have highlighted various chromosomal aberrations in $\mathrm{MB}$, and some of them were found to be associated with clinical outcome. Cytogenetic aberrations in MB involving chromosome 17 as well as amplifications of the $M Y C$ or $M Y C N$ oncogenes have previously been associated with large cell histology and poor patient survival in different studies $[6,7,9,11,13,15$, $17,18,23,24]$. In contrast, several recent reports have identified monosomy of chromosome 6 to be associated with consecutive WNT pathway activation and favorable clinical outcome $[2,8,25]$.

Nevertheless, in contrast to astrocytomas and oligodendrogliomas, molecular progression of MB has only been suspected, but has not been unanimously proven. A stepwise progression of genetic abnormalities in these tumors was identified in a few cases only, when comparing primary and recurrent tumor specimens [3].

Here, we performed comparative histopathologic and cytogenetic analyses of $28 \mathrm{MB}$ cases, for which samples from primary manifestation and tumor recurrence were available. The aim of this study was to determine which histopathological features and chromosome abnormalities may be associated with MB molecular progression over time. Interphase fluorescence in situ hybridization (FISH) was carried out for five regions of interest, namely MYC, MYCN, chromosome 6q, and chromosomal arms $17 \mathrm{p}$ and $17 \mathrm{q}$.

\section{Materials and methods}

Patient population

Specimens from $28 \mathrm{MB}$ patients who experienced disease recurrence were analyzed in this study. All these patients had received reoperations at secondary diagnosis and specimens from primary and subsequent interventions were available for comparative histopathological and molecular analyses. Patient details are outlined in Table 1. There were 21 males and 7 females, and age ranged from 3 to 37 years (median 14 years). Sixteen patients experienced early recurrence $(<4$ years after first diagnosis), and 12 patients had a time to recurrence of 48 months or more (late recurrence). Staging procedures included MRI before and immediately after neurosurgery. Metastatic disease was detected by craniospinal imaging and analysis of the cerebrospinal fluid following the Chang classification. Most of the patients (26) had no leptomeningeal tumor dissemination (i.e. M0 stage) at the time of primary diagnosis. All patients were treated according to a standard treatment protocol, consisting of surgical tumor resection, followed by craniospinal irradiation with $36 \mathrm{~Gy}$ and a boost to the posterior fossa adding up to a total dose of 53-56 Gy, and eight cycles of vincristin, lomustine, and cisplatin. Gross total tumor resection was achieved in 23 patients (82\%) as assessed by early postoperative MRI. Tumors progressed as the following (Table 2): (1) isolated local recurrence (13 cases); (2) local recurrence in combination with leptomeningeal dissemination (six cases); among the latter, in one patient, tumor progressed as local recurrence combined with leptomeningeal dissemination and seedings outside the CNS (bone marrow and soft tissues); (3) isolated leptomeningeal dissemination in nine cases. Specimens from secondary tumors were obtained either after removal of local progression $(n=18)$ or after resection/biopsy of tumor metastases $(n=10)$.

Fluorescence in situ hybridization

Multicolor interphase FISH analysis of 5- $\mu$ m paraffin sections was performed using the following three commercial probe sets delineating the loci of interest (Vysis, USA): (1) dual-color probe set containing a centromere 2p11-q11 probe (spectrum orange) and a locus-specific 2p24/MYCN probe (spectrum green); (2) dual-color probe set containing a centromere $8 \mathrm{p} 11-\mathrm{q} 11$ probe (spectrum green) and a locusspecific 8q24/MYC probe (spectrum orange); (3) multicolor probe set containing locus-specific 17p13.3/LIS1 (spectrum orange), 17q21/RARA (spectrum green), and 6q23/MYB (spectrum aqua) probes. All FISH experiments were performed in duplicate.

Pretreatment of slides, hybridization, posthybridization processing, and signal detection were performed as previously reported $[15,20]$. Briefly, the samples showing sufficient FISH efficiency ( $>90 \%$ nuclei with signals) were evaluated by two independent investigators. Signals were scored in at least 200 nonoverlapping, intact nuclei. 
Table 1 Clinical characteristics of medulloblastoma patients with early progression $(n=16)$ and late progression $(n=12)$

\begin{tabular}{|c|c|c|c|}
\hline Variable & $\begin{array}{l}\text { Early recurrence } \\
\text { ( }<48 \text { months })\end{array}$ & $\begin{array}{l}\text { Late recurrence } \\
\text { ( } \geq 48 \text { months) }\end{array}$ & Total \\
\hline \multicolumn{4}{|l|}{$\mathrm{Age}^{\mathrm{a}}$} \\
\hline Median age (years) & 11.5 & 15 & 14 \\
\hline Range (years) & $3-22$ & $7-37$ & $3-37$ \\
\hline \multicolumn{4}{|l|}{ Gender } \\
\hline Female & $5(31 \%)$ & $2(17 \%)$ & $7(25 \%)$ \\
\hline Male & $11(69 \%)$ & $10(83 \%)$ & $21(75 \%)$ \\
\hline \multicolumn{4}{|l|}{ Chang stage } \\
\hline $\mathrm{M}_{0}$ & $14(87 \%)$ & $12(100 \%)$ & $26(93 \%)$ \\
\hline $\mathrm{M}_{1-3}$ & $2(13 \%)$ & $0(0 \%)$ & $2(7 \%)$ \\
\hline \multicolumn{4}{|l|}{ Level of resection at primary diagnosis } \\
\hline Gross total resection & $12(75 \%)$ & $11(92 \%)$ & \\
\hline Macroscopic tumor residue & $4(25 \%)$ & $1(8 \%)$ & \\
\hline \multicolumn{4}{|l|}{ Type of progression } \\
\hline Local progression & $5(31 \%)$ & $8(67 \%)$ & $13(46 \%)$ \\
\hline Dissemination & $6(38 \%)$ & $3(25 \%)$ & $9(32 \%)$ \\
\hline Local progression and dissemination & $5(31 \%)$ & $1(8 \%)$ & $6(21 \%)$ \\
\hline \multicolumn{4}{|l|}{ Time to progression } \\
\hline Median (months) & 16 & 70 & 34 \\
\hline Range (months) & $4-39$ & $48-138$ & $4-138$ \\
\hline Total & 16 & 12 & 28 \\
\hline
\end{tabular}

${ }^{a}$ At initial diagnosis

MB. The remaining four samples were identified as large

Non-neoplastic cerebellar specimens $(n=10)$ were used as a control for each probe set. Specimens were considered amplified for $M Y C N$ and $M Y C$, when more than $10 \%$ of tumor cells exhibited either more than eight signals of the respective oncogene probe with the reference/control ratio $>2.0$ or innumerable tight clusters of signals of the locusspecific probe. Gains of $6 \mathrm{q}$ and/or $17 \mathrm{q}$ were defined as $>10 \%$ of nuclei containing three or more signals for the respective probes, if no such findings were detected for the $17 \mathrm{p}$ locus (to rule out polyploidy). Hemizygous deletions of $6 q$ and $17 p$ were defined as $>50 \%$ of nuclei (mean \pm three standard deviations in neoplastic controls) containing one signal either for the $17 \mathrm{p}$ or for the $6 \mathrm{q}$ probes and $\geq 2$ signals of the $17 \mathrm{q}$ probe. No homozygous deletions were identified for any of the investigated loci in this study.

\section{Results}

Pathological and clinical findings

On histopathological examination of primary samples, 15 tumors were diagnosed as classic MB without anaplastic features (Table 2). In addition, nine classic MB showed patterns of "mild" anaplasia, according to Eberhart et al. [3, 5]. In this small set of samples, there was no significant difference concerning progression-free survival (PFS) and overall survival (OS) between these two subgroups of classic cell/anaplastic MB. Latter showed significantly shorter median PFS (7 months; $P<0.001)$ compared to classic MB with "mild" anaplasia and those without anaplastic patterns, whereas there were no differences in median PFS for classic MB with "mild" anaplasia and for those without anaplastic patterns (21 and 48 months, respectively; $P=0.78$ ).

Eighteen MB recurrences (14 with classic and 4 with large cell histology) exactly resembled the histopathological features of their corresponding primaries. In contrast, the remaining ten samples with classic histology in the primary tumor clearly showed anaplastic features such as increasing nuclear pleomorphism toward either dark polygonal or pale round nuclei, cell "wrapping," elevated number of mitoses and apoptotic bodies, and appearance of necroses (Fig. 1). In two of these samples, microvascular proliferation was observed in the recurrent tumor. We estimated the cumulative incidence functions from competing risks with respect to the acquisition of such anaplastic patterns. Time to progression for tumors with such acquired anaplastic patterns was longer when compared with samples that showed unchanged histological appearance (Fig. 2).

Cytogenetic aberrations in primary and recurrent tumors

Totally, 84 multicolor FISH hybridizations were performed (Table 2, Fig. 3). Among the primary tumors, 13 samples (46\%) exhibited initially balanced cytogenetic profiles for 
Table 2 Histopathological and molecular characteristics of medulloblastoma patients at primary diagnosis and at the time of disease progression

\begin{tabular}{|c|c|c|c|c|c|c|}
\hline \multirow[t]{2}{*}{ Number } & \multirow{2}{*}{$\begin{array}{l}\text { Time to progression } \\
\text { (months) }\end{array}$} & \multirow{2}{*}{$\begin{array}{l}\text { Type of } \\
\text { progression }\end{array}$} & \multicolumn{2}{|l|}{ Histology } & \multicolumn{2}{|l|}{ Molecular markers } \\
\hline & & & Primary diagnosis & Progression & Primary diagnosis & Progression \\
\hline 1 & 4 & $\mathrm{mts}$ & LCA & LCA & $\mathrm{i} 17 \mathrm{q}$ & $\mathrm{i} 17 \mathrm{q}$ \\
\hline 2 & 6 & Local & LCA & LCA & MYC; i17q; gain 6q & MYC; i17q; gain 60 \\
\hline 3 & 8 & Local $/ \mathrm{mts}$ & LCA & LCA & MYCN; gain $17 \mathrm{q}$ & MYCN; gain $17 \mathrm{q}$ \\
\hline 4 & 8 & $\mathrm{mts}$ & Classic NA & Classic NA & i17q & i17q \\
\hline 5 & 9 & Local $/ \mathrm{mts}$ & Classic NA & Classic NA & $\mathrm{i} 17 \mathrm{q}$ & $\mathrm{i} 17 \mathrm{q}$ \\
\hline 6 & 11 & Local & Classic MA & Classic MA & Balanced & gain $17 q$ \\
\hline 7 & 12 & Local $/ \mathrm{mts}$ & Classic MA & Classic MA & $\mathrm{i} 17 \mathrm{q}$ & $\mathrm{i} 17 \mathrm{q}$ \\
\hline $8^{\mathrm{a}}$ & 15 & Local $/ \mathrm{mts}$ & LCA & LCA & MYC; i17q; gain 6q & MYC; i17q; gain $6 c$ \\
\hline 9 & 17 & Local & Classic MA & Classic MA & Balanced & Balanced \\
\hline 10 & 18 & Local $/ \mathrm{mts}$ & Classic MA & Classic MA & Balanced & gain $17 q$ \\
\hline 11 & 18 & Local & Classic NA & Classic NA & gain $17 q$ & gain $17 q$ \\
\hline 12 & 21 & $\mathrm{mts}$ & Classic MA & Classic MA & MYCN; i17q & i17q \\
\hline 13 & 27 & $\mathrm{mts}$ & Classic NA & Classic NA & MYCN; i17q & i17q \\
\hline 14 & 30 & $\mathrm{mts}$ & Classic NA & Classic MA & $\mathrm{i} 17 \mathrm{q}$ & $\mathrm{i} 17 \mathrm{q}$ \\
\hline 15 & 38 & Local & Classic NA & Classic NA & Balanced & Balanced \\
\hline 16 & 39 & $\mathrm{mts}$ & Classic NA & Classic NA & $\mathrm{i} 17 \mathrm{q}$ & $\mathrm{i} 17 \mathrm{q}$ \\
\hline 17 & 48 & Local & Classic NA & Classic NA & Balanced & Balanced \\
\hline 18 & 57 & Local & Classic MA & LCA & Balanced & MYCN \\
\hline 19 & 58 & $\mathrm{mts}$ & Classic NA & Classic MA & Balanced & balanced \\
\hline 20 & 61 & $\mathrm{mts}$ & Classic MA & LCA & $\mathrm{i} 17 \mathrm{q}$ & i17q \\
\hline 21 & 62 & Local & Classic NA & Classic MA & $\mathrm{i} 17 \mathrm{q}$ & $\mathrm{i} 17 \mathrm{q}$ \\
\hline 22 & 67 & Local & Classic NA & Classic NA & Balanced & Balanced \\
\hline 23 & 73 & $\mathrm{mts}$ & Classic MA & LCA & Balanced & i17q \\
\hline 24 & 78 & Local & Classic NA & Classic MA & Balanced & gain 17q; gain 6q \\
\hline 25 & 91 & Local & Classic NA & LCA & Balanced & MYCN \\
\hline 26 & 96 & Local $/ \mathrm{mts}$ & Classic NA & LCA & Balanced & gain $17 q$; gain $6 q$ \\
\hline 27 & 132 & Local & Classic NA & Classic MA & Balanced & Balanced \\
\hline 28 & 138 & Local & Classic MA & Classic MA & i17q & $\mathrm{i} 17 \mathrm{q}$ \\
\hline
\end{tabular}

Cases with accumulation of histological and molecular changes are indicated in bold

$m t s$ metastatic disease, $L C A$ large cell/anaplastic histology, $N A$ no signs of anaplasia, $M A$ moderate anaplasia

${ }^{\text {a }}$ Case 8 also showed metastasis outside the CNS (soft tissue and bone marrow)

all tested markers, whereas the remaining 15 tumors (54\%) showed one or more aberrations at the loci of interest. Consistent with previously published results, aberrations of chromosome 17 were the most common finding affecting $15(54 \%)$ of the primary tumors. Concomitant loss $17 \mathrm{p}$ and gain $17 \mathrm{q}$ indicative for isochromosome 17 [i(17q)] was detected in 13 cases, while isolated gain of $17 \mathrm{q}$ was found in two tumors. In addition, five samples showed either $M Y C$ or MYCN amplification, and two tumors disclosed 6q gain. There were no tumors with $6 \mathrm{q}$ loss in this series of tumors with recurrence. Comparing cytogenetic findings with histopathological data, we found that 3/4 tumors with large cell histology showed either MYC or MYCN amplifications and all of them carried i(17q). Median OS for patients, whose tumors initially showed genomic aberrations, was shorter than that for patients whose primaries showed balanced profiles (43 and 128 months, respectively, $P=0.18)$. Similar results were obtained for PFS. The estimated median PFS for patients, whose tumors initially showed genomic aberrations, was 18 months, whereas the median PFS for patients whose primaries showed balanced profiles, was 58 months $(P=0.09)$.

Analysis of samples from recurrent tumors disclosed an unchanged cytogenetic profile for the investigated loci in 19 cases (68\%). Among these, six tumors were initially balanced, and 13 samples already showed chromosomal aberrations in their primaries. In the latter 13 cases, we found no differences in the extent of cytogenetic abnormalities when primary and recurrent specimens were compared.

Seven recurrent tumors with initially balanced profile showed appearance of acquired cytogenetic abnormalities involving various loci of interest. Among them, five tumors 
Fig. 1 Examples of increasing anaplasia in recurrent $\mathrm{MB}$ $(H \& E, \times 400)$. a Primary tumor with Homer-Wright pseudorosettes and no signs of anaplasia (Case 24). b Local recurrence of this tumor after 78 months with signs of moderate anaplasia. c Primary tumor with moderate anaplasia (Case 20). d Cranial metastasis of this tumor after 61 months with severe cellular anaplasia. e Primary tumor with signs of focal moderate anaplasia (Case 18). f Local recurrence of this tumor after 57 months with severe diffuse anaplasia
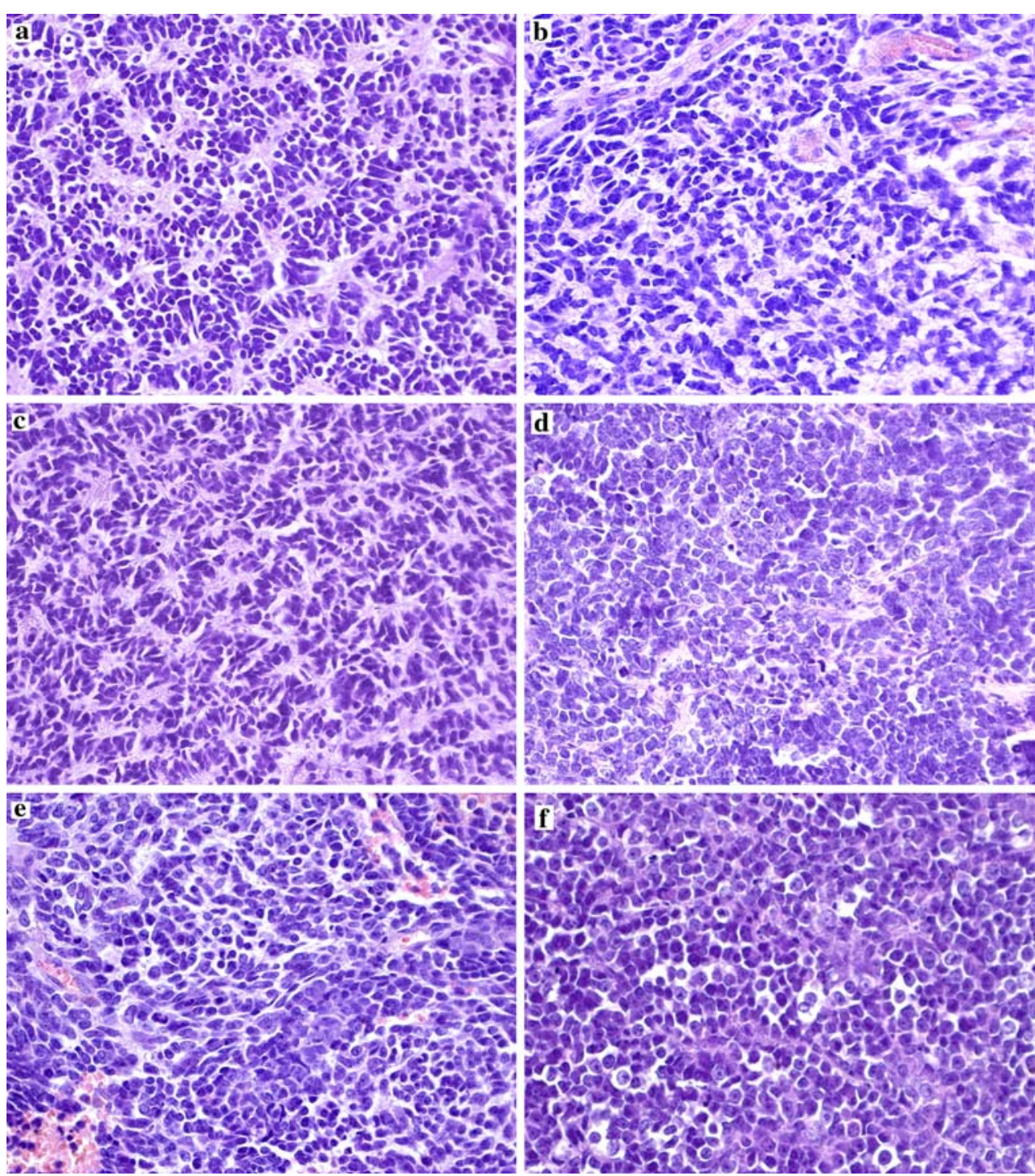

showed acquired abnormalities of 17q accompanied by gain of $6 q$ in two cases. Two other tumors with initially balanced profile disclosed appearance of $M Y C N$ oncogene amplification. Besides this, analysis of two intracranial metastases, which developed from primaries with concomitant MYCN amplification and $\mathrm{i}(17 \mathrm{q})$, revealed that tumor cells bearing oncogene amplification were not detected in secondary lesions, whereas cells with i(17q) were retained.

\section{Discussion}

The concept of anaplastic MB has been expanded for clinical purposes hypothesizing that even a lower degree of anaplasia and/or focal anaplasia may affect patient outcome. Most studies on the role of gradual anaplasia revealed that even focal anaplasia had a significant negative effect on progression-free and overall survival $[3-7,10,13,14]$. However, some other studies showed no association between the degree of anaplasia and MB prognosis [12, 16].

The current concept of the molecular pathogenesis of MB suggests that these tumors have the ability to undergo stepwise progression as it has been demonstrated for other tumors, including neuroectodermal neoplasms [3]. The hypothetic model of MB pathogenesis includes primary "initiation events" (i.e. Hedgehog, NOTCH, and WNT activation) and subsequent "progression events" (i.e. chromosome 17 aberrations, MYC/MYCN amplifications, and others). Nevertheless, this has been a hypothesis only, because conclusive evidence of temporal molecular MB progression has not been presented yet. Only one study reported on $\mathrm{CGH}$ analyses of a single pair of primary $\mathrm{MB}$ and its recurrence [6]. In that case, an increased number of overall chromosomal aberrations was found in the recurrent tumor, one of the acquired lesions being a deletion of $17 \mathrm{p}$.

In the current study investigating 28 pairs of primary $\mathrm{MB}$ and their recurrences, we found an increase in anaplastic 


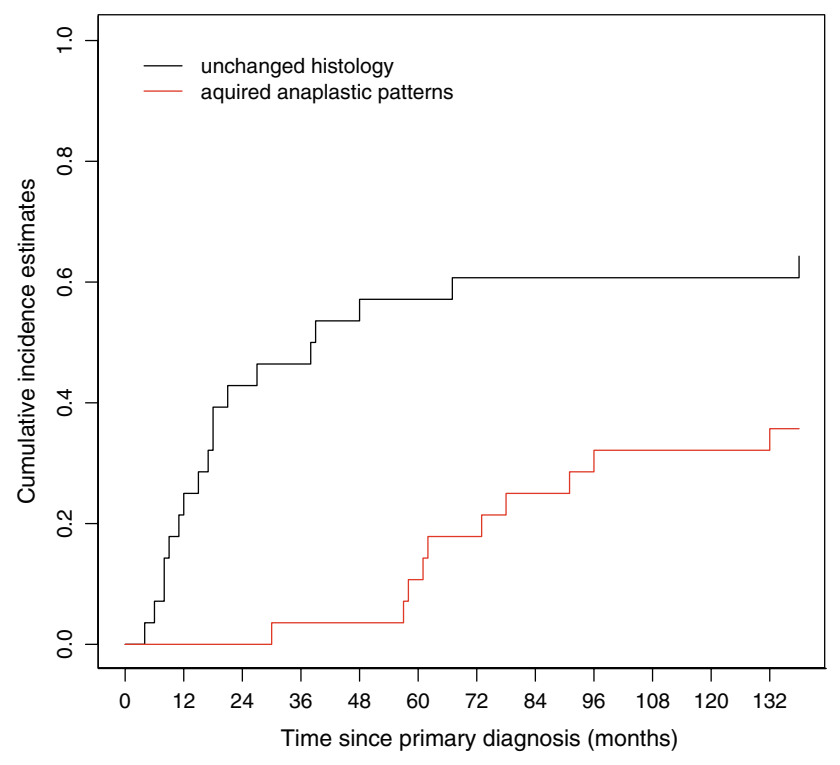

Fig. 2 Cumulative incidence of tumor progression for tumors with acquired anaplastic features in the recurrent tumor and for those without. Time to progression was considerably longer for patients whose recurrent tumors showed acquired anaplastic features

features by histopathological assessment in approximately $40 \%$ of tumors. Notably, the time to progression was longer for tumors with increasing degree of anaplasia
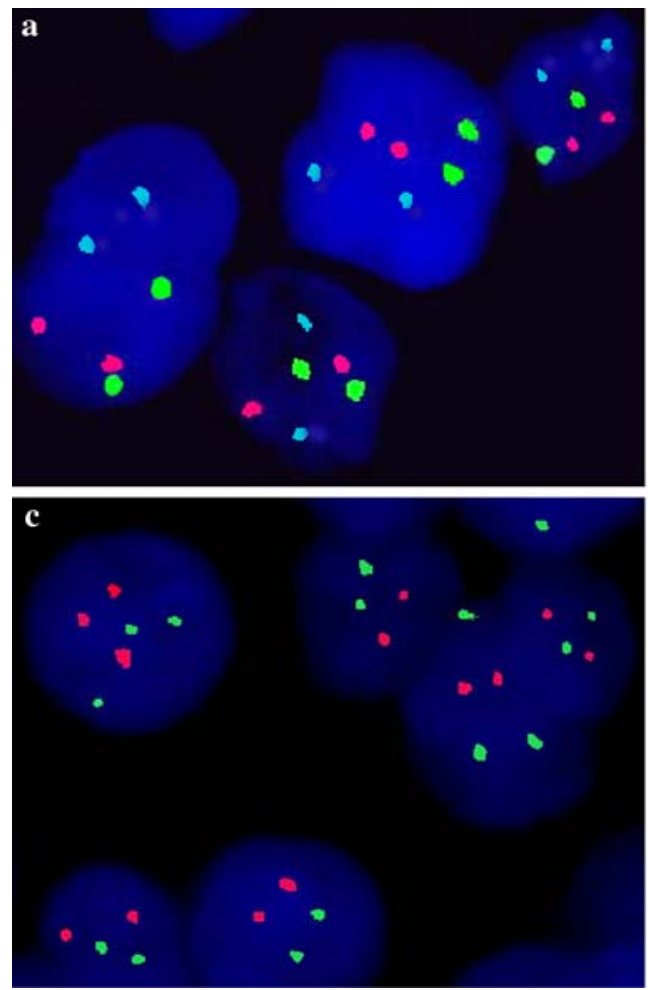

Fig. 3 Acquired cytogenetic aberrations in progressed MB detected by FISH analysis. a Case 26, primary tumor: balanced profile for chromosomal loci 17p13 (red), 17q21 (green), and 6q23 (blue) with two signals for each probe. b Local progression of this tumor after 96 months: appearance of 17q21 and 6q gains (three and more signals). in the recurrence, suggesting that a certain interval is required for gradual accumulation of such changes within the histological appearance. To test the likelihood of molecular progression in $\mathrm{MB}$, we analyzed five chromosomal loci that have been reported to be crucial for MB biology and prognosis $[2,6-9,11,13,15,17,18,23-25]$. We found that patients bearing primary tumors with characteristic chromosomal aberrations such as $M Y C$ or $M Y C N$ oncogene amplification, $17 \mathrm{q}$ gain, or $6 \mathrm{q}$ gain had a shorter median PFS and OS, suggesting that tumor cells with these chromosomal abnormalities represent cytological sources for selective growth advantage underlying further clinical progression per se. These findings are exactly in line with our results obtained from larger series of MB [19].

MYCN amplification is a hallmark of tumors with poor clinical outcome [13, 19]. Intriguingly, two MB showed unexpected loss of cells bearing $M Y C N$ amplification within their intracranial metastatic deposits. Metastatic lesions have been widely thought to arise from a small selected fraction of cells in the primary tumor, which share molecular alterations associated with loss of cell adhesion and increased cell motility. Perhaps, $M Y C N$ amplification has no effects on the capability of MB cells to disseminate throughout the CNS. However, this alteration seems to induce locally aggressive behavior, as we found acquired


c Case 25, primary tumor: balanced profile for chromosomal loci 2p24/ MYCN (green) and 2p11-q11 (red) with two signals for each probe. d Local progression of this tumor after 91 months: appearance of $M Y C N$ oncogene amplification (numerous green signals and signal clusters) 
MYCN amplification in two local MB recurrences that were removed 5 and 7.5 years after the primary operation, respectively. To our knowledge, this is the first report on postponed oncogene amplifications in MB progression. Interestingly, both these tumors showed local tumor progression, but no signs of metastatic disease (Table 2). Undoubtedly, the analysis of two cases only allows for careful general conclusions. Nevertheless, these findings appear to be intriguing and further analysis of genetic alterations associated with MB dissemination is warranted.

Five of 13 tumors (38\%), whose primaries disclosed balanced profiles for all investigated loci, showed acquired anomalies of chromosome 17, accompanied by gains of $6 \mathrm{q}$ in two tumors. Notably, cases with isolated gain of $17 \mathrm{q}$ (without concomitant deletion of $17 \mathrm{p}$ ) prevailed in this group (four out of five), whereas $i(17 q)$ comprised the vast majority of abnormalities detected in primary tumors (13 out of 15 tumors). These findings strongly support the notion that $\mathrm{i}(17 \mathrm{q})$ may represent a unique primary chromosomal aberration in a subset of $\mathrm{MB}[3,15,18]$. On the contrary, isolated gain of chromosome $17 \mathrm{q}$ appears to be a centrally important aberration for the development of metastatic disease. This is in line with our recent demonstration of isolated gain of $17 \mathrm{q}$ being associated with unfavorable prognosis, whereas isolated $17 \mathrm{p}$ deletion is not [19].

In general, all our findings indicate that sequential chromosomal aberrations of the type seen in other neuroectodermal tumors can occur in MB as well. Nevertheless, it remains uncertain whether these cytogenetic abnormalities are already intrinsically present in a small (and accordingly "invisible") fraction of primary tumor cells with their sequential clonal expansion after treatment or accumulated over time in a stepwise cytogenetic fashion.

In conclusion, we found that MB can undergo temporal molecular progression with accumulation of "prognostically unfavorable" cytogenetic changes, including even acquisition of oncogene amplifications. Thus, treatment failures after standard combined treatment of MB patients may, at least in part, be caused by acquired and steady progressing changes in tumor biology, especially in tumors showing late recurrence. Particularly, when considering targeted therapy approaches for relapsed patients, secondary biopsy should be performed to assess the biologic properties of the recurrent tumor, which may differ substantially from the primary tumor.

Acknowledgments This study was supported by a grant from the Deutsche Kinderkrebsstiftung to S. Pfister.

Open Access This article is distributed under the terms of the Creative Commons Attribution Noncommercial License which permits any noncommercial use, distribution, and reproduction in any medium, provided the original author(s) and source are credited.

\section{References}

1. Albright A, Wisoff J, Zeltzer P, Boyett J, Rorke L, Stanley P (1996) Effects of medulloblastoma resections on outcome in children: a report from the Children's Cancer Group. Neurosurgery 38(2):265-271. doi:10.1097/00006123-199602000-00007

2. Clifford S, Lusher M, Lindsey J et al (2006) Wnt/wingless pathway activation and chromosome 6 loss characterise a distinct molecular sub-group of medulloblastomas associated with a favourable prognosis. Cell Cycle 5(22):2666-2670

3. Eberhart C, Burger P (2003) Anaplasia and grading in medulloblastomas. Brain Pathol 13(3):376-385

4. Eberhart C, Cohen K, Tihan T, Goldthwaite P, Burger P (2003) Medulloblastomas with systemic metastases: evaluation of tumor histopathology and clinical behavior in 23 patients. J Pediatr Hematol Oncol 25(3):198-203. doi:10.1097/00043426-20030300000004

5. Eberhart C, Kepner J, Goldthwaite P et al (2002) Histopathologic grading of medulloblastomas: a Pediatric Oncology Group study. Cancer 94(2):552-560. doi:10.1002/cncr.10189

6. Eberhart C, Kratz J, Schuster A et al (2002) Comparative genomic hybridization detects an increased number of chromosomal alterations in large cell/anaplastic medulloblastomas. Brain Pathol 12(1):36-44

7. Eberhart C, Kratz J, Wang Y et al (2004) Histopathological and molecular prognostic markers in medulloblastoma: c-myc, N-myc, TrkC, and anaplasia. J Neuropathol Exp Neurol 63(5):441-449

8. Ellison DW, Onilude OE, Lindsey JC et al (2005) ß-Catenin status predicts a favorable outcome in childhood medulloblastoma: the United Kingdom Children's Cancer Study Group Brain Tumour Committee. J Clin Oncol 23(31):7951-7957. doi:10.1200/ JCO.2005.01.5479

9. Gajjar A, Hernan R, Kocak M et al (2004) Clinical, histopathologic, and molecular markers of prognosis: toward a new disease risk stratification system for medulloblastoma. J Clin Oncol 22(6):984 993. doi:10.1200/JCO.2004.06.032

10. Giangaspero F, Wellek S, Masuoka J, Gessi M, Kleihues P, Ohgaki H (2006) Stratification of medulloblastoma on the basis of histopathological grading. Acta Neuropathol 112(1):5-12. doi:10.1007/s00401-006-0064-x

11. Gilbertson R, Wickramasinghe C, Hernan R et al (2001) Clinical and molecular stratification of disease risk in medulloblastoma. $\mathrm{Br}$ J Cancer 85(5):705-712. doi:10.1054/bjoc.2001.1987

12. Giordana M, D'Agostino C, Pollo B et al (2005) Anaplasia is rare and does not influence prognosis in adult medulloblastoma. J Neuropathol Exp Neurol 64(10):869-874

13. Lamont JM, McManamy CS, Pearson AD, Clifford SC, Ellison DW (2004) Combined histopathological and molecular cytogenetic stratification of medulloblastoma patients. Clin Cancer Res 10(16):5482-5493. doi:10.1158/1078-0432.CCR-03-0721

14. McManamy C, Lamont J, Taylor R et al (2003) Morphophenotypic variation predicts clinical behavior in childhood non-desmoplastic medulloblastomas. J Neuropathol Exp Neurol 62(6):627632

15. Mendrzyk F, Radlwimmer B, Joos S et al (2005) Genomic and protein expression profiling identifies CDK6 as novel independent prognostic marker in medulloblastoma. J Clin Oncol 23(34):88538862. doi:10.1200/JCO.2005.02.8589

16. Min H, Lee Y, Park K, Cho B, Park S (2006) Medulloblastoma: histopathologic and molecular markers of anaplasia and biologic behavior. Acta Neuropathol 112(1):13-20. doi:10.1007/s00401006-0073-9

17. Neben K, Korshunov A, Benner A et al (2004) Microarray-based screening for molecular markers in medulloblastoma revealed 
STK15 as independent predictor for survival. Cancer Res 64(9):3103-3111. doi:10.1158/0008-5472.CAN-03-3968

18. Pan E, Pellarin M, Holmes E et al (2005) Isochromosome $17 \mathrm{q}$ is a negative prognostic factor in poor-risk childhood medulloblastoma patients. Clin Cancer Res 11(13):4733-4740. doi:10.1158/ 1078-0432.CCR-04-0465

19. Pfister S, Remke M, Benner A, et al (2008) Molecular risk stratification of pediatric medulloblastoma based on DNA copy-number aberrations of chromosomes $6 \mathrm{q}, 17 \mathrm{q}$, and the MYC/MYCN loci. $\mathrm{J}$ Clin Oncol (in revision)

20. Pfister S, Remke M, Toedt G et al (2007) Supratentorial primitive neuroectodermal tumors of the central nervous system frequently harbor deletions of the CDKN2A locus and other genomic aberrations distinct from medulloblastomas. Genes Chromosomes Cancer 46(9):839-851. doi:10.1002/gcc.20471

21. Polkinghorn W, Tarbell N (2007) Medulloblastoma: tumorigenesis, current clinical paradigm, and efforts to improve risk stratification. Nat Clin Pract Oncol 4(5):295-304. doi:10.1038/ncponc0794

22. Ray A, Ho M, Ma J et al (2004) A clinicobiological model predicting survival in medulloblastoma. Clin Cancer Res 10(22):76137620. doi:10.1158/1078-0432.CCR-04-0499
23. Rutkowski S, von Bueren A, von Hoff K et al (2007) Prognostic relevance of clinical and biological risk factors in childhood medulloblastoma: results of patients treated in the prospective multicenter trial HIT'91. Clin Cancer Res 13(9):2651-2657. doi:10.1158/1078-0432.CCR-06-1779

24. Scheurlen WG, Schwabe GC, Joos S, Mollenhauer J, Sorensen N, Kuhl J (1998) Molecular analysis of childhood primitive neuroectodermal tumors defines markers associated with poor outcome. J Clin Oncol 16(7):2478-2485

25. Thompson MC, Fuller C, Hogg TL et al (2006) Genomics identifies medulloblastoma subgroups that are enriched for specific genetic alterations. J Clin Oncol 24(12):1924-1931. doi:10.1200/ JCO.2005.04.4974

26. Zeltzer PM, Boyett JM, Finlay JL et al (1999) Metastasis stage, adjuvant treatment, and residual tumor are prognostic factors for medulloblastoma in children: conclusions from the Children's Cancer Group 921 randomized phase III study. J Clin Oncol 17(3):832-845

27. Zerbini C, Gelber R, Weinberg D et al (1993) Prognostic factors in medulloblastoma, including DNA ploidy. J Clin Oncol 11(4):616622 